\title{
Q\&A: How do plants respond to cytokinins and what is their importance?
}

\author{
Asami Osugi ${ }^{1}$ and Hitoshi Sakakibara ${ }^{1,2^{*}}$
}

\begin{abstract}
Cytokinins comprise a family of signaling molecules essential for regulating the growth and development of plants, acting both locally and at a distance. Although much is known about their biosynthesis and transport, important open questions remain.
\end{abstract}

\section{What are cytokinins?}

Cytokinins are a well-studied family of plant hormones. In the 1950s, Skoog and Miller first determined the structural formula of a cytokinin, 6-furfurylaminopurine, a substance isolated from a herring sperm DNA preparation that promotes plant cell division in vitro. It was named kinetin (Fig. 1). However, 6-furfurylaminopurine is artificially formed under specific conditions, such as when DNA preparations degrade or are autoclaved, and was not known to occur naturally. Subsequently, $N^{6}-\left(\Delta^{2}\right.$-isopentenyl)adenine (iP), trans-zeatin (tZ), ciszeatin (cZ), dihydrozeatin, and topolins were identified as natural cytokinins in planta (Fig. 1a) [1]. iP and tZ play a major physiological role in Arabidopsis and many other plant species due to higher relative abundance and affinity to their receptors. Some plants, including major crops (for example, maize and rice), contain $\mathrm{cZ}$ and its conjugates as the most abundant cytokinin species (Fig. 1b). Even in these plants, iP and $\mathrm{tZ}$ assume a central role for cytokinin actions, while the physiological significance of $\mathrm{cZ}$ has not been fully elucidated. As shown in Fig. 1, cytokinins include various chemicals, but natural cytokinins commonly contain an adenine moiety and a side chain modification at the adenine N6 position. Natural and artificial cytokinins are recognized by common cytokinin receptors [2].

\footnotetext{
* Correspondence: sakaki@riken.jp

'RIKEN Center for Sustainable Resource Science, 1-7-22, Suehiro, Tsurumi, Yokohama 230-0045, Japan

${ }^{2}$ Graduate School of Bioagricultural Sciences, Nagoya University, Chikusa, Nagoya 464-8601, Japan
}

\section{What are the physiological functions of cytokinins?}

Cytokinins were originally defined as chemicals that induce cell proliferation and trigger callus differentiation to shoot when applied with auxins, but now it is known that cytokinins play a key role in many aspects of plant growth and development [3], including embryogenesis, maintenance of root and shoot meristems, and vascular development. They also modulate root elongation, lateral root number, nodule formation, and apical dominance in response to environmental stimuli. Thus, cytokinins are important signaling molecules for regulating growth and development throughout the life of the plant.

\section{What is the physiological concentration of cytokinins?}

The recent development of mass spectrometry technology has enabled us to quantify the concentration of phytohormones and their conjugates at the organ level. Based on such analyses, for which the values are generally 0.1 to $10 \mathrm{pmol} \mathrm{g}^{-1}$ fresh weight, it may be estimated that the in vivo concentration of cytokinins is at the nanomolar level, and that concentration may vary between different organs and growth conditions. Notably, if cytokinins are unevenly distributed in the organs at either the tissue or cellular level, their local concentration could be higher or lower. The affinity (apparent $K_{\mathrm{D}}$ ) of cytokinin receptors to their ligands is around 1-40 nM [4], which would make the estimated nanomolar concentration of cytokinins physiologically relevant.

\section{How do plants sense cytokinins?}

Cytokinins mainly trigger physiological responses through the regulation of gene expression. A two-component system (TCS) is employed to transduce the cytokinin signal to the target genes. The TCS, which typically consists of a sensory histidine kinase and response regulator, was first discovered in prokaryotes, where it serves to enable response to environmental stimuli. Since its first mention in 1996 [5], extensive biochemical and genetic studies have 


\section{A}<smiles>CC(C)=CCNc1ncnc2[nH]cnc12</smiles>

iP

$\mathrm{HO}$<smiles>CC(=CCNc1ncnc2[nH]cnc12)CO</smiles>

tZ

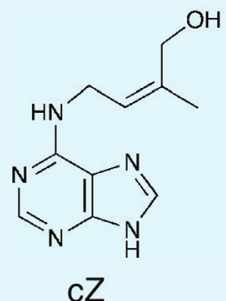<smiles>Oc1ccccc1CNc1ncnc2[nH]cnc12</smiles>

oT<smiles>CC(CO)CCNc1ncnc2[nH]cnc12</smiles>

DZ

\section{Artificial CKs}<smiles>c1coc(CNc2ncnc3[nH]cnc23)c1</smiles>

Kinetin<smiles>O=C(Nc1ccccc1)Nc1cnns1</smiles>

TDZ
B
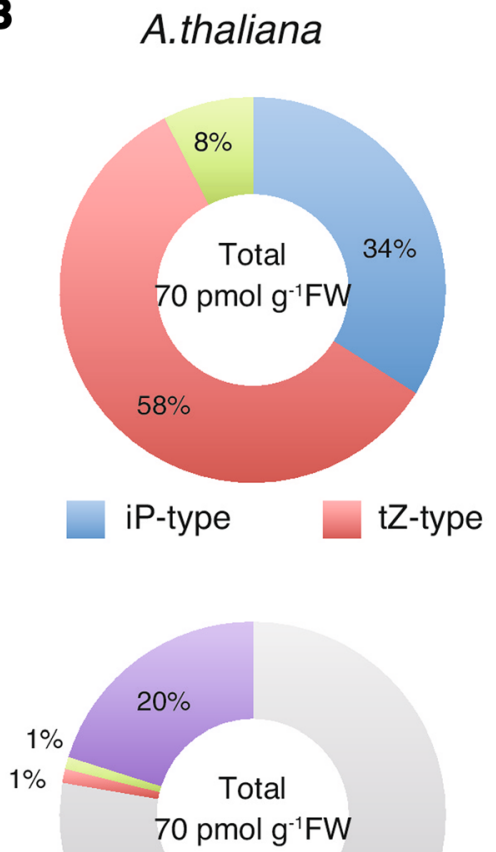

$78 \%$
O. sativa

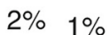

Total $420 \mathrm{pmol} \mathrm{g}^{-1} \mathrm{FW}$

$97 \%$

cZ-type

$0.4 \% \quad 3 \%$

Total

$420 \mathrm{pmol} \mathrm{g}^{-1} \mathrm{FW}$

$95 \%$

CK CK-ribosides CK-ribotides CK-glucosides

Fig. 1. (See legend on next page.) 
(See figure on previous page.)

Fig. 1. Structure and composition of cytokinins. a Structures of various cytokinins (CKs). $N^{6}$-( $\Delta^{2}$-isopentenyl)adenine (iP), trans-zeatin (tZ), cis-zeatin $(C Z)$, dihydrozeatin (DZ), and ortho-topolin (OT) are shown as representative natural CKs. Kinetin and thidiazuron (TDZ) may activate cytokinin receptors when administered, but are not physiological regulators of plant growth. $\mathbf{b}$ Pie charts showing relative abundance of cytokinin species in shoots of Arabidopsis thaliana (left) and Oryza sativa (right). The upper charts show side chain-variant breakdown: $t Z$ and its conjugates (tZ-type), iP and its conjugates (iP-type), and cZ and its conjugates (cZ-type) cytokinins. The lower charts show conjugate-variant breakdown: active form (CK), ribosides (CK-ribosides), ribotides (CK-ribotides), and glucosides (CK-glucosides). Calculations are based on typical quantification data from [31] and [28] for Arabidopsis and rice, respectively. FW fresh weight

been conducted with Arabidopsis to identify and characterize the cytokinin TCS. Today, it is widely accepted that the TCS of cytokinin signaling consists of three groups of proteins in Arabidopsis: three histidine kinases (AHKs; AHK2, AHK3 and AHK4/WOL1/CRE1), five histidine-containing phosphotransfer proteins (AHPs; AHP1-AHP5) and eleven type-B response regulators (type-B ARRs; ARR1, ARR2, ARR10-ARR14 and ARR18ARR21) (Fig. 2) [3].

AHKs are membrane-localized cytokinin receptors which consist of three domains: CHASE, histidine kinase, and receiver $[2,3,6]$. The binding of cytokinins to the AHK CHASE domain triggers autophosphorylation of a His residue in its histidine kinase domain, and the phosphoryl group is internally transferred to an Asp residue in its receiver domain. Recent studies suggest that the cytokinin receptors are localized in both the plasma membrane and the endoplasmic reticulum $[7,8]$, but it is still unclear whether both are functional or not.

AHPs mediate the transfer of a phosphoryl group from cytosolic AHKs to nuclear-localized type-B ARRs [3, 9].
Type-B ARRs possess a receiver domain for the phosphoryl group, a DNA-binding domain (GARP domain), and a glutamine-rich domain for transcriptional activation [10]. Transcriptional activation by ARRs is repressed in the non-phosphorylated state [10]. When phosphorylated, type-B ARRs can bind target DNA sequences and activate transcription of target genes.

Since each member of the AHK, AHP and type-B ARR gene families is functionally redundant, clear phenotypic differences are not observed in their single mutants. However, responsiveness to cytokinins is severely reduced and growth phenotypes could be observed in multiple mutants within each gene family [11-13], indicating that the cytokinin TCS plays a central role in cytokinin responses in plants.

\section{How does the TCS modulate its signaling flux?}

In order to finely regulate cytokinin signaling, multiple feedback loops of the cytokinin TCS are employed to ensure the appropriate signaling flux (Fig. 2). In addition to the 11 type-B ARRs, the Arabidopsis genome has ten

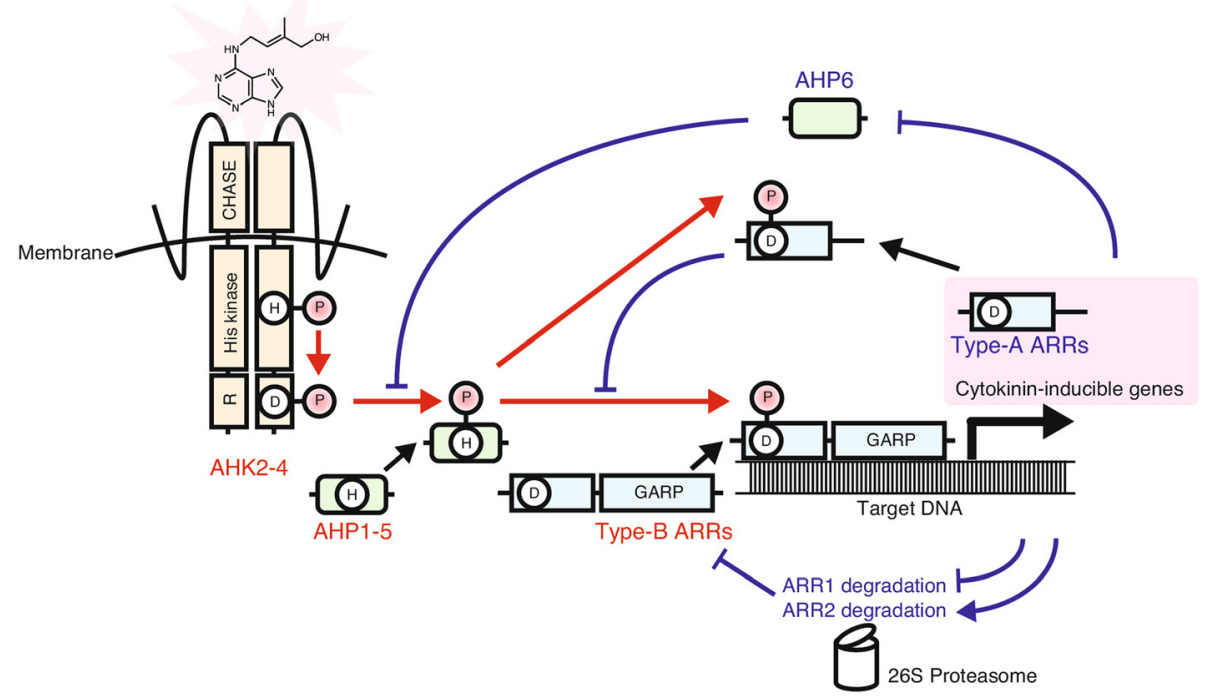

Fig. 2. Diagram of the cytokinin two component system (TCS). AHKS (AHK2, AHK3 and AHK4/WOL1/CRE1) are autophosphorylated in response to cytokinins. The phosphoryl group is transferred to type-B ARRs through AHPs. Phosphorylated type-B ARRs bind to target DNA and induce the expression of a set of genes involved in cytokinin primary response. The stability of type-A ARRs, which repress cytokinin TCS signaling, is controlled through proteolysis by the 265 proteasome in a feedback loop. Expression of AHP6, which inhibits phosphotransfer between AHKs and canonical AHPs, is repressed by cytokinin. Red arrows indicate phosphotransfer. Blue solid arrows and T-end lines represent positive and negative regulation, respectively 
type-A ARR genes (ARR3-ARR9, ARR15-ARR17) [3], and some of them are direct targets of type-B ARRs. Like type-B ARRs, type-A ARRs possess a receiver domain and receive a phosphoryl group from AHPs, but they lack a DNA-binding domain. Thus, type-A ARRs can potentially inhibit the cytokinin signaling flux by competing with type-B ARRs for phosphate transfer [14].

Differential regulation of posttranslational stability of type-B ARRs is involved in the modulation of the TCS signaling flux. Cytokinins promote degradation of ARR2 via the $26 \mathrm{~S}$ proteasome [15] while they stabilize ARR1 by preventing degradation by $26 \mathrm{~S}$ proteasome [16]. On the other hand, KISS ME DEADLY proteins (KMDs), a family of F-box proteins, are involved in degrading ARR1, ARR2 and ARR12, but the detailed mechanisms have yet to be discovered [17].

It has also been reported that an AHP homologue, AHP6, which lacks the conserved histidine for phosphoryl group transfer, physically interacts with AHKs but does not receive a phosphate, suggesting that AHP6 inhibits cytokinin response through competition with canonical AHPs. Cytokinins repress AHP6 expression [18], which suggests that the promotion of cytokinin signaling flux is in part mediated by the down-regulation of AHP6. It was reported that cell-specific expression of AHP6 serves spatial specification of cytokinin signaling $[19,20]$. In addition, cytokinins induce the expression of a cytokinin receptor gene, $A H K 4 / W O L 1 / C R E 1$, which might lead to increased sensitivity to cytokinins [21]. It has been proposed that occurrence of these feedback loops in an organ- or a cell-specific manner is important for regulation of cytokinin signaling flux, as described later.

\section{How are cytokinins produced and metabolized in plants?}

Cytokinin levels in vivo are determined by the balance between biosynthesis and catabolism. Biosynthesis of cytokinins is regulated by three key enzymes (Fig. 3). First, adenosine-phosphate isopentenyltransferase (IPT) catalyzes the formation of iP ribonucleotides from dimethylallyl diphosphate (DMAPP) and adenine nucleotides, with preferential use of ATP or ADP [22, 23]. Cytochrome P450 monooxygenase 735A (CYP735A) hydroxylates the end of the prenyl side chain of iP ribonucleotides, with a preference for iP-riboside 5'-monophosphate (iPRMP) or diphosphate (iPRDP), to produce tZ ribotides [24]. Finally, LONELY GUY (LOG), a phosphoribohydrolase, converts the precursors iPRMP and tZ-riboside 5'-monophosphate (tZRMP) to their active forms, iP and $t Z$, respectively [25]. Although cytokinin ribonucleosides, such as iP ribonucleoside and $\mathrm{tZ}$ ribonucleoside, are widely found in plant tissues, they are considered to be precursors and mainly converted to their active form via LOG after phosphorylation, because $\log$ septuple mutants, in which the LOG- dependent pathway is impaired, exhibit severe cytokinindeficient phenotypes in Arabidopsis [26]. The biosynthesis of cytokinins is regulated through IPT genes by various internal and external environmental stimuli, such as nitrogen sources [27, 28], and other phytohormones [29].

For the catabolism of cytokinins, cytokinin oxidase/ dehydrogenase (CKX) irreversibly degrades cytokinins by cleaving the unsaturated isoprenoid side chain, which results in the formation of adenine and the corresponding aldehyde [3]. Glycosylation of cytokinins also plays an important role in modulating cytokinin activity: $N$-glucosylation of the N7 and N9 positions of the adenine moiety and $O$-glucosylation of the zeatin side chain are catalyzed by UGT76C1 and $\mathrm{C} 2$, and $85 \mathrm{~A} 1$, respectively, in Arabidopsis [3, 30]. Because of their biological stability, the glucosides are the most abundant components of cytokinin derivatives, making up $80 \%$ or more of total cytokinin-related compounds in plants (Fig. 1b). These glucosides are thought to be sequestered in the vacuole. In addition, ribosidation and ribotidation by purine salvage pathway enzymes contribute to the cycling of cytokinins, through reverting active forms back to inactive precursors (Fig. 3). Thus, homeostasis of cytokinin activity is maintained by multiple metabolic systems.

\section{Where are cytokinins produced in plants?}

Cytokinins are more abundant in developing tissues and organs, such as root tip, shoot apex, cambium, and immature organs, and initially it was thought that cytokinins are synthesized in these limited tissues and organs. However, recent studies provide us with insight into cytokininproducing and -degrading sites. There are seven IPT genes (IPT1 and IPT3-IPT8), two CYP735A genes (CYP735A1 and $A 2$ ), seven functional $L O G$ genes (LOG1-LOG5, LOG7 and LOG8) and seven CKX genes (AtCKX1AtCKX7) in Arabidopsis [3]. These gene families show various expression patterns $[27,29,31-33]$. Notably, IPT3 is expressed in the phloem in both roots and aerial organs, suggesting that the precursor of iP-type cytokinins could be synthesized in a wide range of plant parts [27, 29] (Fig. 4). On the other hand, CYP735A is predominantly expressed in root vasculature [31]. Such spatial distribution of IPT gene and CYP735A expression causes the preferential synthesis of $\mathrm{tZ}$ in roots and of iP in shoots. Expression of the $L O G$ gene family covers almost all organs. Thus, the activation step of cytokinin synthesis can occur wherever the LOG genes are expressed [32]. These expression patterns suggest that cytokinins play roles in both long-distance and local signaling.

\section{How important are locally produced cytokinins for plant development?}

The importance of cytokinins' local action is best characterized by their role in maintenance of shoot apical 


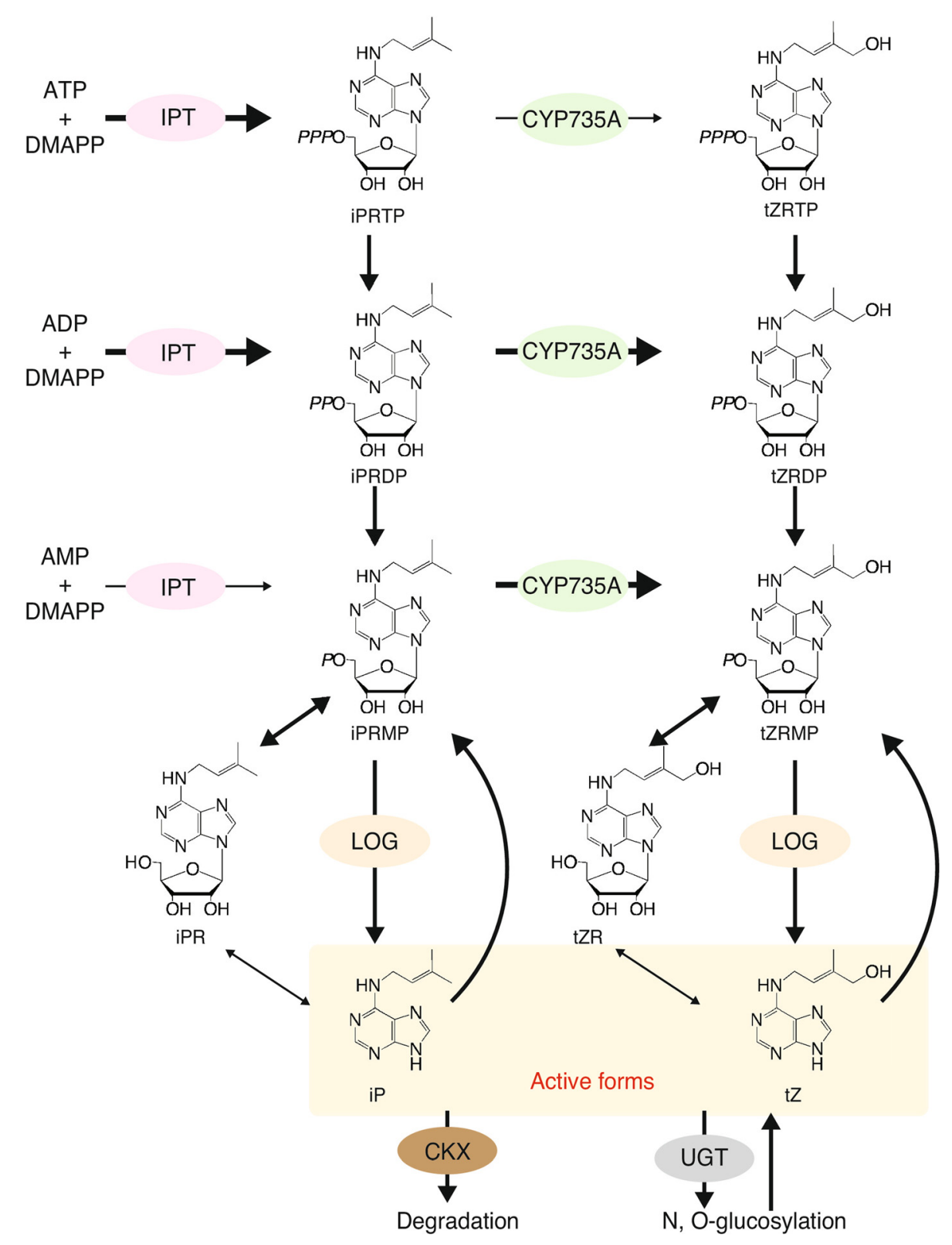

Fig. 3. Current model of iP and tZ biosynthesis and metabolic pathways in Arabidopsis. In Arabidopsis, IPT preferentially utilizes ATP and ADP, and CYP735A preferentially utilizes IPRMP and iPRDP, as substrates. LOG exclusively reacts with their monophosphate forms. Active cytokinins are degraded by CKX, glucosylated by UGT, or reverted to their precursors by the purine salvage pathway

meristem and root vascular development (Fig. 5). In Oryza sativa (rice), a single log mutation confers reduced shoot apical meristem size and abnormal floral organ development, although ten LOG-like genes are expressed in various organs [25]. Similarly, although the genes of the Arabidopsis LOG family are expressed in various types of tissues and organs and thought to be functionally redundant [32], the single $\log 7$ mutation causes reduction of shoot apical meristem size [26]. Rice LOG and Arabidopsis LOG7 are expressed in a restricted region of the upper part of the shoot apical meristem.
Thus, loss of function of certain LOG genes expressed in this specific site is not fully complemented by other LOG genes expressed in other parts of the plant. These facts indicate that maintenance of shoot apical meristem activity requires site-specific activation of cytokinins.

In order to maintain shoot apical meristem, it is necessary to channel the cytokinin signal towards the organizing center [34]. In shoot apical meristem, active cytokinins are produced in the L1 layer by LOG4 and in the central zone by LOG7 [35, 36]. The organizing center and rib meristem are responsive to cytokinins via 


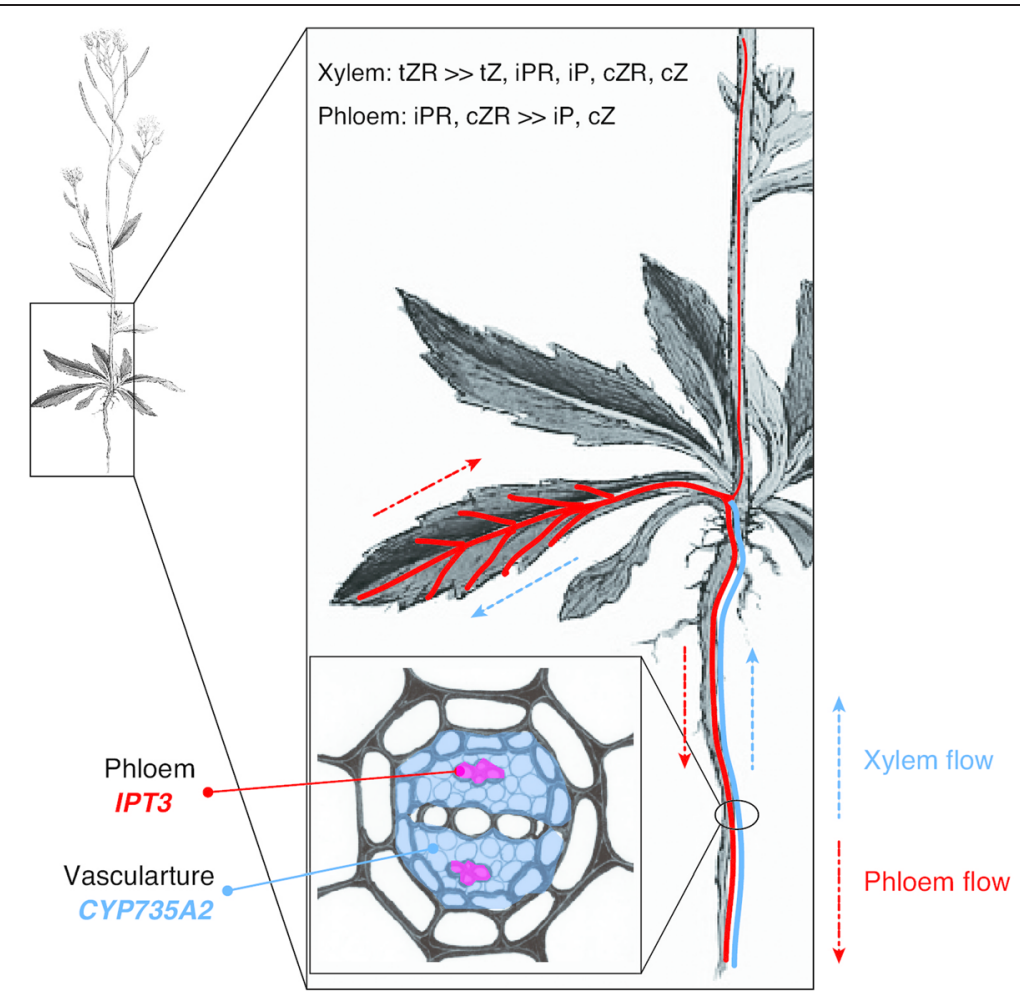

Fig. 4. Spatial expression patterns of IPT3 and CYP735A2 in Arabidopsis. Spatial expression patterns of IPT3 and CYP735A2 are indicated in red and blue, respectively. IPT3 is predominantly expressed in phloem, and CYP735A2 in root vasculature. tZR is the major form of xylem cytokinins, and iPR and CZR are found in phloem [40], suggesting that iP-type and tZ-type cytokinins are directionally translocated between organs. The Arabidopsis picture is modified from Sowerby et al. [53]

AHK4/WOL1/CRE1 (Fig. 5a) [36]. Endogenous cytokinins activate cytokinin signaling only in the organizing center but not in the rib meristem, although exogenously applied cytokinins activate both of these tissues (Fig. 5a) [36]. This indicates that the longitudinal diffusion of cytokinins to adjacent regions and local action in the restricted cells are important for proper meristem function.

The importance of local activation of cytokinins is also shown in the early stage of root vascular development. In root tip vasculature, which consists of procambium, protoxylem, metaxylem and phloem (Fig. 5b), cytokinin-

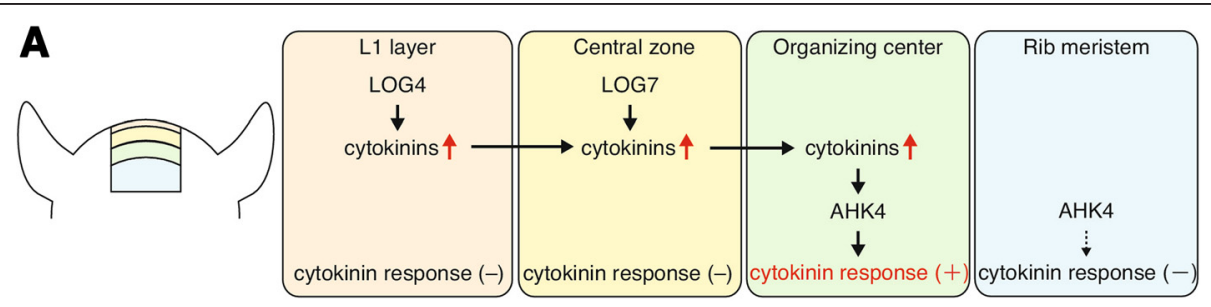

B

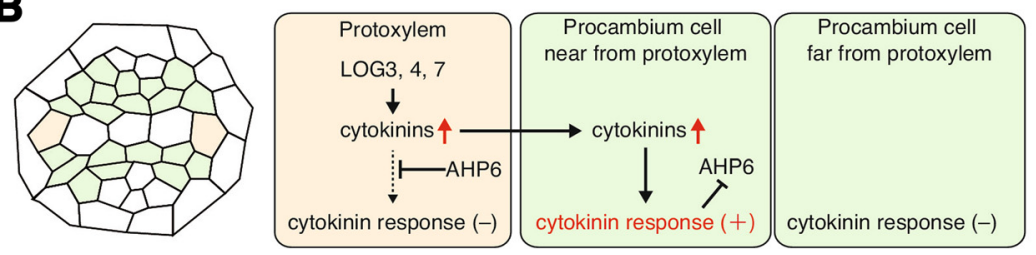

Fig. 5. Local function of cytokinins in shoot apical meristem and root tip. a Schematic diagram of cytokinin production and response in shoot apical meristem. Active cytokinins are produced in the epidermal L1 layer and central zone. They diffuse basipetally and function in the AHK4-expressing organizing center. $\mathbf{b}$ Schematic diagram of cytokinin production and response in root tip. Active cytokinins are produced in AHP6-expressing protoxylem. They diffuse and function in adjacent procambium cells while repressing AHPG expression 
activating LOG3 and LOG4 are specifically expressed in protoxylem cells, while cytokinin-perceiving $A H K 4 /$ WOL1/CRE1 is expressed in the procambium $[19,20]$. Analysis using marker genes for cytokinin response revealed that procambium cells adjacent to protoxylem respond to cytokinins more strongly than distant procambium cells $[19,20]$, indicating that local activation of cytokinins in protoxylem specifies cytokinin perception cells in procambium. Since cytokinins inhibit procambium-to-protoxylem differentiation, this regulation may be necessary for proper positioning of those cells during vascular development.

Root morphogenesis is abnormal in the $\log 3 \log 4 \log 7$ triple mutant, possibly due to reduced levels of active cytokinin [19]. Expression of LOG3 under a xylem precursor cell-specific promoter rescues this phenotype. However, ectopic expression of LOG3 in the phloem, through which cytokinins are systemically transported, does not. Therefore, it is suggested that the locally synthesized cytokinins are necessary for root morphogenesis.

\section{How important are distantly translocated cytokinins for plant growth and development?}

Movement of cytokinins between organs has been shown by tracer experiments using isotope-labeled cytokinins [31, 37-39] and detection of cytokinins in vascular saps also supports translocation of cytokinins [40]. In general, long-distance signals are translocated through xylem and phloem, two major conduits for material transfer in plant vasculature. Intriguingly, these studies show that cytokinin species are unevenly distributed: tZ-type cytokinins are more abundant than iP-type in xylem sap and vice versa in phloem sap [40] (Fig. 4). These studies imply that iP-type and tZ-type cytokinins are directionally translocated and transmit different biological messages between organs.

Grafting experiments support the importance of cytokinins as long-distance signals. The Arabidopsis ipt1 ipt3 ipt5 ipt7 quadruple mutant shows severely reduced cytokinin content and shoot and root growth-deficient phenotypes [41]. Reciprocal grafting between the quadruple mutant and wild type rescued the growth-deficient phenotypes in shoot and root with accompanying recovery of cytokinin content [41], indicating a role of root-borne cytokinins in shoot and vice versa.

Grafting experiments also show the importance of root-borne $\mathrm{tZ}$ for normal shoot growth. In the cyp $735 a 1$ cyp735a2 double mutant, tZ-type cytokinin content is severely reduced without affecting total cytokinin quantity, and shoot growth is retarded [31]. When the shoot of the double mutant is grafted onto wild-type stock, the shoot phenotype is complemented with accompanying recovery of tZ-type cytokinin content, suggesting that side chain modulation of cytokinins has a specialized role in their long-distance translocation for shoot growth regulation.

As for the role of shoot-borne cytokinin in roots, impairing phloem transport destabilizes root vascular development with an accompanying reduction of basipetal cytokinin translocation in Arabidopsis [37]. Thus, shoot-borne cytokinins, via phloem, could participate in normal development of root vasculature in coordination with locally produced cytokinins.

In Lotus japonicas, root nodule number is reduced in the Ljipt3 cytokinin biosynthesis gene mutant [38], as well as in a Ljipt3 knockdown transgenic line [42]. Grafting between Ljipt3 shoot and wild-type root stock represses nodulation, while reverse grafting does not [38]. This suggests that shoot-derived cytokinins function as a repression signal of nodulation. It is notable that cytokinins synthesized in the root are not significantly involved in regulation of nodule number, although the mechanism through which the origin of cytokinins is determined remains to be elucidated.

So far, studies have shown that both xylem and phloem sap contain vast amounts of cytokinin ribonucleosides [40], suggesting that cytokinin ribonucleosides are translocated through the vasculature. It is believed that the cytokinin ribonucleosides are converted to their ribonucleotides followed by activation via LOG where they function.

\section{How is long-distance translocation of cytokinins regulated?}

An elaborate translocation system is necessary for the regulation of organ-to-organ communication via cytokinins. Recent studies identified ATP-binding cassette transporter subfamily G14 (ABCG14) as a key gene for appropriate root-to-shoot cytokinin translocation [39, 43]. In abcg14, tZ-type cytokinin contents are greatly reduced in xylem sap, and the dwarf phenotype of abcg14 is rescued in grafted plants between $a b c g 14$ shoot and wildtype root stock [43], indicating that $A B C G 14$ is an essential gene for root-to-shoot translocation of cytokinins. Since the biochemical properties of ABCG14 have not been well characterized, the substrate of ABCG14 has not been identified. In addition to ABCG14, purine permease 1 and 2 (PUP1 and PUP2) and equilibrate nucleoside transporter $(E N T)$ have been shown in in vitro studies to be involved in transport of cytokinins $[44,45]$. However, their functions in planta, especially in long-distance translocation of cytokinins, are still poorly characterized.

\section{What determines the site of cytokinin action?}

Spatial regulation of LOG expression is one of the determining factors that specify the functional sites of cytokinins, and expression of each LOG family gene is regulated in a site-specific manner $[25,32]$. Recently, a 
mechanism for this site-specific expression of LOG genes has been reported in flower development in Arabidopsis [46]. In a mutant of APETALA1 (AP1), a MADS-BOX transcriptional factor, abnormal floral organs are observed. AP1 directly represses LOG1 expression, and possibly in sepals where their expression overlaps. LOG1 repression, under control of the $A P 1$ promoter, partially rescued the ap1 phenotype, indicating that sepal-specific LOG1 repression is required for normal flower development. In addition, expression of a $L O G$ homolog is directly activated by KNOTTED1 (KN1) in maize [47]. It is thought that KN1 might provide site-specific regulation of the LOG homolog's coordination with certain BEL1-LIKE $H O M E O B O X(B L H)$ gene products, which interact with KN1 to bind DNA.

Recent studies have also revealed the importance of cytokinin oxidase/dehydrogenase (CKX) function as a metabolic attenuator of local cytokinin action. As well as $L O G$, the expression of each $C K X$ family gene is regulated in a site-specific manner [33]. Loss-of-function mutants of specific CKX genes in rice (OsCKX2) and Arabidopsis (CKX3 and $C K X 5)$, which are expressed in reproductive meristem, cause increased cytokinin levels, leading to larger meristem size and an increase in reproductive organ number $[48,49]$, clearly showing that the CKXs fine-tune active cytokinin levels at the expression site. $C K X 3$ expression in the organizing center and CKX5 broadly in the meristematic domain [49] may be particularly important for ensuring the site-specific cytokinin response (Fig. 5a). It is also intriguing that Arabidopsis AP1, which negatively regulates $L O G 1$ expression, positively regulates $C K X 3$ in the sepal [46]. This regulation is important for development of a determinate floral meristem.

Another candidate to control functionality of cytokinins is the family of AHK proteins. AHK promoter:reporter gene analyses revealed that the cytokinin receptors are expressed in various tissues in the plant, but that $A H K 3$ is preferentially expressed in aerial organs, such as rosette leaves, AHK4/WOL1/CRE1 is expressed in root, and $A H K 2$ is expressed in both [11]. Interestingly, it is reported that their affinities to ligands are different: $A H K 2$ and AHK4/WOL1/CRE1 bind with similar affinity to $\mathrm{tZ}$ as well as iP, while $A H K 3$ has less affinity to iP than to $\mathrm{tZ}[4,50]$. Thus, it is expected that $\mathrm{tZ}$ plays a major role as a cytokinin in shoots. This supports previous work with cyp $735 a 1$ cyp $735 a 2$ identifying a specialized function of $\mathrm{tZ}$ in shoot development [31].

Some other components of the cytokinin TCS also contribute to determining the sites of action of cytokinins. Cytokinins induce expression of WUSCHEL (WUS), a transcriptional factor expressed in the organizing center of the shoot apical meristem. WUS directly binds to the promoters of ARR5, ARR6, ARR7, and ARR15, and represses their expression (Fig. 5) [34]. This regulation establishes a local spatial domain for the organization of a stem cell niche in the shoot apex. (Fig. 5) [51].

\section{What interactions occur between cytokinins and other phytohormones?}

Signaling systems of phytohormones build a network and mutually regulate signaling, transport, and metabolic systems. Interplay between cytokinins and auxin is one of the best-characterized cases of hormone-hormone interaction. Recent studies shed light on the importance of cytokinin-auxin interaction for auxin traffic and specification of cytokinin action sites in root vascular development. Cytokinin signaling in the procambium up-regulates expression of PINs, a family of auxin efflux carriers, and promotes their distribution in the plasma membrane from anticlinal to periclinal [52]. The bisymmetric distribution of PINs channels basipetally translocates auxin to protoxylem via the procambium. The auxin is perceived in the protoxylem and induces LONESOME HIGHWAY (LHW) and TARGET OF MONOPTEROS5 (TMO5). They directly induce LOG3 and LOG4, which activate cytokinins, and $A H P 6$, which inhibits cytokinin responses in the protoxylem $[19,20]$. Thus, the auxin-induced LOG3 and LOG4 in protoxylem provides cytokinins to adjacent procambium for proper root vascular development while the induced AHP6 inhibits cytokinin response (Fig. 5b) [19, 20]. Consequently, the procambial cytokinin response regulates PIN expression and distribution.

\section{What is the important question for the future?}

Cytokinins positively regulate agriculturally important traits such as grain size and biomass [3] but they also promote unfavorable phenotypes such as inhibition of root elongation. Therefore, spatio-temporal regulation of cytokinins is required for appropriate function in specific organs. In this article, we have discussed the spatial regulation of cytokinin biosynthesis and the role of these hormones in signaling. In order to better utilize cytokinin action to enhance beneficial traits of crops, a deeper understanding of their temporal regulation will be necessary.

\section{Competing interests}

The authors declare that they have no competing interests.

\section{Acknowledgements}

The authors are supported by MEXT NC-CARP and JST CREST.

Published online: 27 November 2015

References

1. Sakakibara H. Cytokinins: activity, biosynthesis and translocation. Annu Rev Plant Biol. 2006;57:431-49.

2. Yamada H, Suzuki T, Terada K, Takei K, Ishikawa K, Miwa K, et al. The Arabidopsis AHK4 histidine kinase is a cytokinin-binding receptor that transduces cytokinin signals across the membrane. Plant Cell Physiol. 2001;42:1017-23.

3. Kieber JJ, Schaller GE. Cytokinins. In The Arabidopsis book. American Society of Plant Biologists; 2014:12. 
4. Lomin SN, Krivosheev DM, Steklov MY, Arkhipov DV, Osolodkin DI, Schmülling T, et al. Plant membrane assays with cytokinin receptors underpin the unique role of free cytokinin bases as biologically active ligands. J Exp Bot. 2015. doi:10.1093/jxb/eru522.

5. Kakimoto T. CKI1, a histidine kinase homolog implicated in cytokinin signal transduction. Science. 1996;274:982-5.

6. Inoue T, Higuchi M, Hashimoto Y, Seki M, Kobayashi M, Kato T, et al. Identification of CRE1 as a cytokinin receptor from Arabidopsis. Nature. 2001;409:1060-3.

7. Lomin SN, Yonekura-Sakakibara K, Romanov GA, Sakakibara H. Ligand-binding properties and subcellular localization of maize cytokinin receptors. J Exp Bot. 2011;62:5149-59.

8. Wulfetange K, Lomin SN, Romanov GA, Stolz A, Heyl A, Schmülling T. The cytokinin receptors of Arabidopsis are located mainly to the endoplasmic reticulum. Plant Physiol. 2011;156:1808-18.

9. Hwang I, Sheen J. Two-component circuitry in Arabidopsis cytokinin signal transduction. Nature. 2001:413:383-9.

10. Sakai H, Honma T, Aoyama T, Sato S, Kato T, Tabata S, et al. ARR1, a transcription factor for genes immediately responsive to cytokinins. Science. 2001;294:1519-21.

11. Higuchi M, Pischke MS, Mähönen AP, Miyawaki K, Hashimoto $Y$, Seki M, et al. In planta functions of the Arabidopsis cytokinin receptor family. Proc Natl Acad Sci U S A. 2004;101:8821-6.

12. Mason MG, Mathews DE, Argyros DA, Maxwell BB, Kieber JJ, Alonso JM, et al. Multiple type-B response regulators mediate cytokinin signal transduction in Arabidopsis. Plant Cell. 2005;17:3007-18.

13. Hutchison CE, Li J, Argueso C, Gonzalez M, Lee E, Lewis MW, et al. The Arabidopsis histidine phosphotransfer proteins are redundant positive regulators of cytokinin signaling. Plant Cell. 2006;18:3073-87.

14. Kiba T, Yamada H, Sato S, Kato T, Tabata S, Yamashino T, et al. The type-A response regulator, ARR15, acts as a negative regulator in the cytokininmediated signal transduction in Arabidopsis thaliana. Plant Cell Physiol. 2003:44:868-74.

15. Kim K, Ryu H, Cho Y-H, Scacchi E, Sabatini S, Hwang I. Cytokinin-facilitated proteolysis of Arabidopsis response regulator 2 attenuates signaling output in two-component circuitry. Plant J. 2012;69:934-45.

16. Kurepa J, Li Y, Smalle JA. Cytokinin signaling stabilizes the response activator ARR1. Plant J. 2014;78:157-68.

17. Kim HJ, Chiang Y-H, Kieber JJ, Schaller GE. ${ }^{\mathrm{scf}}$ KMD controls cytokinin signaling by regulating the degradation of type-B response regulators. Proc Natl Acad Sci U S A. 2013;110:10028-33.

18. Mähönen AP, Bishopp A, Higuchi M, Nieminen KM, Kinoshita K, Törmäkangas K, et al. Cytokinin signaling and its inhibitor AHP6 regulate cell fate during vascular development. Science. 2006:311:94-8.

19. Ohashi-Ito K, Saegusa M, Iwamoto K, Oda Y, Katayama H, Kojima M, et al. A bHLH complex activates vascular cell division via cytokinin action in root apical meristem. Curr Biol. 2014;24:2053-8.

20. De Rybel B, Adibi M, Breda AS, Wendrich JR, Smit ME, Novák O, et al. Integration of growth and patterning during vascular tissue formation in Arabidopsis. Science. 2014;345:1255215.

21. Kiba T, Aoki K, Sakakibara H, Mizuno T. Arabidopsis response regulator, ARR22, ectopic expression of which results in phenotypes similar to the wol cytokinin-receptor mutant. Plant Cell Physiol. 2004;45:1063-77.

22. Kakimoto T. Identification of plant cytokinin biosynthetic enzymes as dimethylallyl diphosphate: ATP/ADP isopentenyltransferases. Plant Cell Physiol. 2001;42:677-85.

23. Takei K, Sakakibara H, Sugiyama T. Identification of genes encoding adenylate isopentenyltransferase, a cytokinin biosynthesis enzyme, in Arabidopsis thaliana. J Biol Chem. 2001;276:26405-10.

24. Takei K, Yamaya T, Sakakibara H. Arabidopsis CYP735A1 and CYP735A2 encode cytokinin hydroxylases that catalyze the biosynthesis of trans-zeatin. J Biol Chem. 2004;279:41866-72.

25. Kurakawa T, Ueda N, Maekawa M, Kobayashi K, Kojima M, Nagato Y, et al. Direct control of shoot meristem activity by a cytokinin-activating enzyme. Nature. 2007:445:652-5.

26. Tokunaga H, Kojima M, Kuroha T, Ishida T, Sugimoto K, Kiba T, et al. Arabidopsis lonely guy (LOG) multiple mutants reveal a central role of the LOG-dependent pathway in cytokinin activation. Plant J. 2012;69:355-65.

27. Takei K, Ueda N, Aoki K, Kuromori T, Hirayama T, Shinozaki K, et al. AtIPT3 is a key determinant of nitrate-dependent cytokinin biosynthesis in Arabidopsis. Plant Cell Physiol. 2004;45:1053-62.
28. Kamada-Nobusada T, Makita N, Kojima M, Sakakibara H. Nitrogen-dependent regulation of de novo cytokinin biosynthesis in rice: the role of glutamine metabolism as an additional signal. Plant Cell Physiol. 2013;127:1881-93.

29. Miyawaki K, Matsumoto-Kitano M, Kakimoto T. Expression of cytokinin biosynthetic isopentenyltransferase genes in Arabidopsis: tissue specificity and regulation by auxin, cytokinin, and nitrate. Plant J. 2004;37:128-38.

30. Wang J, Ma XM, Kojima M, Sakakibara H, Hou BK. N-glucosyltransferase UGT76C2 is involved in cytokinin homeostasis and cytokinin response in Arabidopsis thaliana. Plant Cell Physiol. 2011;52:2200-1.

31. Kiba T, Takei K, Kojima M, Sakakibara H. Side-chain modification of cytokinins controls shoot growth in Arabidopsis. Dev Cell. 2013;27:452-61.

32. Kuroha $T$, Tokunaga $H$, Kojima M, Ueda N, Ishida T, Nagawa S, et al. Functional analyses of lonely guy cytokinin-activating enzymes reveal the importance of the direct activation pathway in Arabidopsis. Plant Cell. 2009;21:3152-69.

33. Werner T, Motyka V, Laucou V, Smets R, Van Onckelen H, Schmülling T. Cytokinin-deficient transgenic Arabidopsis plants show multiple developmental alterations indicating opposite functions of cytokinins in the regulation of shoot and root meristem activity. Plant Cell. 2003;15:2532-50.

34. Leibfried A, To JP, Busch W, Stehling S, Kehle A, Demar M, et al. WUSCHEL controls meristem function by direct regulation of cytokinin-inducible response regulators. Nature. 2005;438:1172-5.

35. Yadav RK, Girke T, Pasala S, Xie M, Reddy GV. Gene expression map of the Arabidopsis shoot apical meristem stem cell niche. Proc Natl Acad Sci U S A. 2009;106:4941-6.

36. Chickarmane VS, Gordon SP, Tarr PT, Heisler MG, Meyerowitz EM. Cytokinin signaling as a positional cue for patterning the apical-basal axis of the growing Arabidopsis shoot meristem. Proc Natl Acad Sci U S A. 2012;109:4002-7.

37. Bishopp A, Lehesranta S, Vatén A, Help H, El-Showk S, Scheres B, et al. Phloem-transported cytokinin regulates polar auxin transport and maintains vascular pattern in the root meristem. Curr Biol. 2011;21:927-32.

38. Sasaki T, Suzaki T, Soyano T, Kojima M, Sakakibara H, Kawaguchi M. Shoot-derived cytokinins systemically regulate root nodulation. Nat Commun. 2014;5:4983.

39. Zhang K, Novak O, Wei Z, Gou M, Zhang X, Yu Y, et al. Arabidopsis ABCG14 protein controls the acropetal translocation of root-synthesized cytokinins. Nat Commun. 2014:5:3274

40. Hirose N, Takei K, Kuroha T, Kamada-Nobusada T, Hayashi H, Sakakibara H. Regulation of cytokinin biosynthesis, compartmentalization and translocation. J Exp Bot. 2008;59:75-83.

41. Matsumoto-Kitano M, Kusumoto T, Tarkowski P, Kinoshita-Tsujimura K, Václavíková K, Miyawaki K, et al. Cytokinins are central regulators of cambial activity. Proc Natl Acad Sci U S A. 2008;105:20027-31.

42. Chen Y, Chen W, Li X, Jiang H, Wu P, Xia K, et al. Knockdown of LjIPT3 influences nodule development in Lotus japonicus. Plant Cell Physiol. 2013;55:183-93.

43. Ko D, Kang J, Kiba T, Park J, Kojima M, Do J, et al. Arabidopsis ABCG14 is essential for the root-to-shoot translocation of cytokinin. Proc Natl Acad Sci U S A. 2014;111:7150-5.

44. Burkle L, Cedzich A, Dopke C, Stransky H, Okumoto S, Gillissen B, et al. Transport of cytokinins mediated by purine transporters of the PUP family expressed in phloem, hydathodes, and pollen of Arabidopsis. Plant J. 2003;34:13-26.

45. Hirose N, Makita N, Yamaya T, Sakakibara H. Functional characterization and expression analysis of a gene, OsENT2, encoding an equilibrative nucleoside transporter in Oryza sativa suggest a function in cytokinin transport. Plant Physiol. 2005;138:196-206.

46. Han Y, Zhang C, Yang H, Jiao Y. Cytokinin pathway mediates APETALA1 function in the establishment of determinate floral meristems in Arabidopsis. Proc Natl Acad Sci U S A. 2014;111:6840-5.

47. Bolduc N, Yilmaz A, Mejia-Guerra MK, Morohashi K, O'Connor D, Grotewold E, et al. Unraveling the KNOTTED1 regulatory network in maize meristems. Genes Dev. 2012;26:1685-90.

48. Ashikari M, Sakakibara H, Lin S, Yamamoto T, Takashi T, Nishimura A, et al. Cytokinin oxidase regulates rice grain production. Science. 2005;309:741-5.

49. Bartrina I, Otto E, Strnad M, Werner T, Schmülling T. Cytokinin regulates the activity of reproductive meristems, flower organ size, ovule formation, and thus seed yield in Arabidopsis thaliana. Plant Cell. 2011;23:69-80.

50. Stolz A, Riefler M, Lomin SN, Achazi K, Romanov GA, Schmülling T. The specificity of cytokinin signalling in Arabidopsis thaliana is mediated by differing ligand affinities and expression profiles of the receptors. Plant J. 2011;67:157-68. 
51. Gordon SP, Chickarmane VS, Ohno C, Meyerowitz EM. Multiple feedback loops through cytokinin signaling control stem cell number within the Arabidopsis shoot meristem. Proc Natl Acad Sci U S A. 2009;106:16529-34.

52. Bishopp A, Help H, El-Showk S, Weijers D, Scheres B, Friml J, et al. A mutually inhibitory interaction between auxin and cytokinin specifies vascular pattern in roots. Curr Biol. 2011;21:917-26.

53. Sowerby J, Boswell JT, Lankester P, Salter JW, de C Sowerby J, Sowerby JE. English botany, or coloured figures of British plants, vol. 3. London:

R Hardwicke; 1864. 